\title{
Nachweis von Zuckern aus Glykoproteiden des Magensaftes bei normaler Magenschleimhaut und chronischer Gastritis
}

\author{
Von G. Berg, H. D. Groben, K. Heinkel und N. Hennịng
}

Aus der Medizinischen Klinik der Universität Erlangen-Nürnberg (Direktor: Prof. Dr. N. Henning)

(Eingegangen am 22. Juli 1965)

\begin{abstract}
Mit der Hochspannungselektrophorese wurden bei der Trennung der Zuckerkomponenten des Magenleersekretes keine zufriedenstellenden Ergebnisse erzielt. Die Hydrolyse mit saurem Ionenaustauscher lieferte günstigere Ergebnisse als die Säurehydrolyse. Papierchromatographisch konnten Galaktosamin, Glukosamin, Galaktose, Glukose, Mannose und Fukose nachgewiesen werden. Glukuronsäure ist mit der beschriebenen Methode nicht darzustellen. Bei 9 Patienten mit normaler Magenschleimhaut und 13 Kranken mit Gastritis zeigte sich, daß die einzelnen Zuckerkomponenten qualitativ und quantitativ in keinerlei Beziehung zum histologischen Schleimhautbefund ștanden. Eine gewisse Ausnahme machen Aminozucker, da sich statistisch eine Tendenz des höheren relativen Prozentgehaltes bei chronischer Gastritis nachweisen ließ:

High voltage electrophoresis was applied to the separation of the sugat components of the secretion from fasted stomach, but the results were unsatisfactory. Hydrolysis with an acidic ion exchanger gave more favourable results than acid hydrolysis. Galactosamine, glucosamine, galactose, glucose, mannose and fucose were shown by paper chromatography. Glucuronic acid cannot be isolated by the described method. In 9 patients with a normal gastric mucosa and 13 patients with gastritis, it was shown that the quantity and type of the individual sugar components was completely unrelated to histological differences in the gastric mucosa. The amino sugars are an exception, since, statistically, their relative percentage content tends to be higher in chronic gastritis.
\end{abstract}

Glykoproteide sind ein wichtiger Bestandteil des Magensaftes. Nach früheren Untersuchungen ist, ähnlich wie in anderen serösen und mukösen menschlichen Sekreten, das Eiweiß/Kohlenhydrat-Verhältnis im Vergleich zum Serum stark zugunsten der Kohlenhydrate verschoben $(1,2,3)$. Bei diffusen Magenschleimhautveränderungen unterschieden sich die von der Schleimhaut produzierten Sekrete hinsichtlich ihres Glykoproteidgehaltes und hinsichtlich des Musters der Zuckerbausteinanteile $(1,2)$. Ziel der vorliegenden Untersuchungen war es, in Fortsetzung der bisherigen Arbeiten, zu prüfen, ob das Muster der Zuckerbausteine bei Gesunden und Magenkranken unterschiedlich ist, wobei als Bezugssystem der morphologische Befund der Magenschleimhaut gewählt wurde.

\section{Methodik}

Gewinnung und Hydrolyse des Magensaftes

Magenleersekret von 12 nicht ausgewählten Patienten wurde durch eine dünnkalibrige Verweilsonde gewonnen. Der Nüchterninhalt wurde verworfen, das speichelfreie Sekret durch Aspiration im Abstand von 10-15 Minuten gesammelt. Um den sichtbaren Schleim schneller abzutrennen, wurde 10 Minuten bei 10000 U./Min. zentrifugiert. Bei einem Teil der Patienten wurde der Magensaft mit einem sauren Ionenaustauscher (Permutit RS, Verhältnis Ionenaustauscher/Magensaft 1:2) 48 Stunden bei $100^{\circ}$, beim anderen Teil mit $4 n$ Salzsäure (Verhältnis Magensaft/Salzsäure 1:2) 2 Stunden bei $100^{\circ}$ hydrolysiert. Nach vollzogener Hydrolyse wurde der mit dem Ionenaustauscher behandelte Magensaft mit Wasser und einem Methanol/Äthanol-Gemisch ausgewaschen. Die Neutralisation erfolgte durch Abdestillieren der Säure; der fast bis zur Trockene eingeengte Rückstand wurde in einem Äthanol/Butanol-Gemisch aufgenommen.

Magensaftanalyse mittels Hochspannungselektrophorese

Zur Analyse der Zuckerkomponenten des Mágensafthydrolysates wurde der Pherograph "Original-Frankfurt" nach WIELANDPfleiderer ${ }^{1}$ ) benutzt. Die Trennung erfolgte auf dem Papier „Ederol Nr. 208“, Schleicher-Schüll 2045 M, unter Verwendung eines Boratpuffers $\mathrm{pH}$ 9,6 (Boratpuffergemisch 6,2 $+240 \mathrm{~m} / 1 n$ 1) Hersteller: Fa. L. Hormuth, Inh. W. E. Vetter, Wissenschaftliche Apparate Heidelberg-Wiesloch.
$\mathrm{NaOH}$ ad $1000 \mathrm{~m} /$ aqua dest.) (4). Die Auftragstellen des Hydrolysates wurden mit dem zum Vergleich aufgebrachten Testzuckergemisch nach Vorversuchen in das kathodennahe Drittel des Trennbogens gelegt. Das Testzuckergemisch enthielt Galaktosamin, Glukosamin, Galaktose, Glukose, Mannose, Fukose und Glukuronsäure. Das Hydrolysat wurde bei einer Trennzeit von 1,5 Stunden bei einer Spannung von $1750 \mathrm{~V}$ getrennt. Anschließend wurde der Papierbogen hitzegetrocknet. Der Nachweis der einzelnen Zucker erfolgte mit Anilinphthalat bzw. Ninhydrin $(5,6)$.

\section{Magensaftanalyse mittels Papierchromato- graphie}

Das Leersekret von 22 Patienten mit bekanntem, durch Saugbiopsie ermittelten histologischen Magenschleimhautbefund wurde, wie oben angegeben, aufgearbeitet. Bei den chromatographischen Untersuchungen wurde die Hydrolyse mit einem sauren Ionenaustauscher (Permutit RS, Verhältnis Ionenaustauscher/Magensaft $1: 2$ ) über 48 Stunden bei $100^{\circ}$ vorgenommen, weil dieses Verfahren schonender erschien und weniger Umwandlungsprodukte der Zucker auftraten. Nach der Hydrolyse wurde der Ionenaustaüscher ebenfalls mit Wasser und einem Methanol/Åthanol-Gemisch ausgewaschen. Bei einem Teil des Materials wurde der Magensaft mit einer bestimmten Menge Aktivkohle zur Reinigung und damit zur besseren Differenzierung der einzelnen Zuckerkomponenten versetzt und anschließend die Kohle abfiltriert. Durch $\mathrm{Zu}-$ satż von Alkohol wurde erreicht, daß die Zuckerfraktionen nicht von der Kohle adsorbiert wurden. Die weitere Aufbereitung des Magensaftes effolgte wie oben dargelegt. Der fertig zubereitete Magensaft wurde auf Papierstreifen („Ederol Nr.208“, SchleicherSchüll $2045 \mathrm{M}$ ) von $3,3 \times 30 \mathrm{~cm}$ basisnahe strichförmig aufgetragen. Die Auftragsmenge entsprach etwa 3,3 bzw. 6,6 m/nativem Magensaft. Butanol/Eisessig/Wasser im Verhältnis $4: 1: 1$ wurde für den ersten Durchlauf als Laufmittel für die aufsteigende Chromatographie verwendet, für den zweiten Durchlauf Butanol/Pyridin/ Wasser im Verhältnis 6:4:3. Die zweimalige Chromatographie erschien notwendig, um eine bessere Differenzierung der einzelnen Zuckerkomponenten zu erreichen. Nach der chromatographischen Trennung wurden die Streifen getrocknet, die reduzierenden Zucker mit Anilinphthalat, die Aminozucker mit Ninhydrin und p-Dimethylaminobenzaldehyd-Acetylaceton im Sprühverfahren angefärbt $\left.{ }^{2}\right)(5,6)$. Mittels der Elson-Morgan- und der Ninhy-

2) Die speziellen Zuckernachweise für Glukose und Galaktose wurden dankenswerterweise von Herrn Privatdozent Dr. HorsTmanN und Herrn Dr. Frscher, Physiol.-Chem. Institut der Universität Erlangen-Nürnberg (Direktor: Prof. Dr. Dr. F. MAY) vorgenommen. 


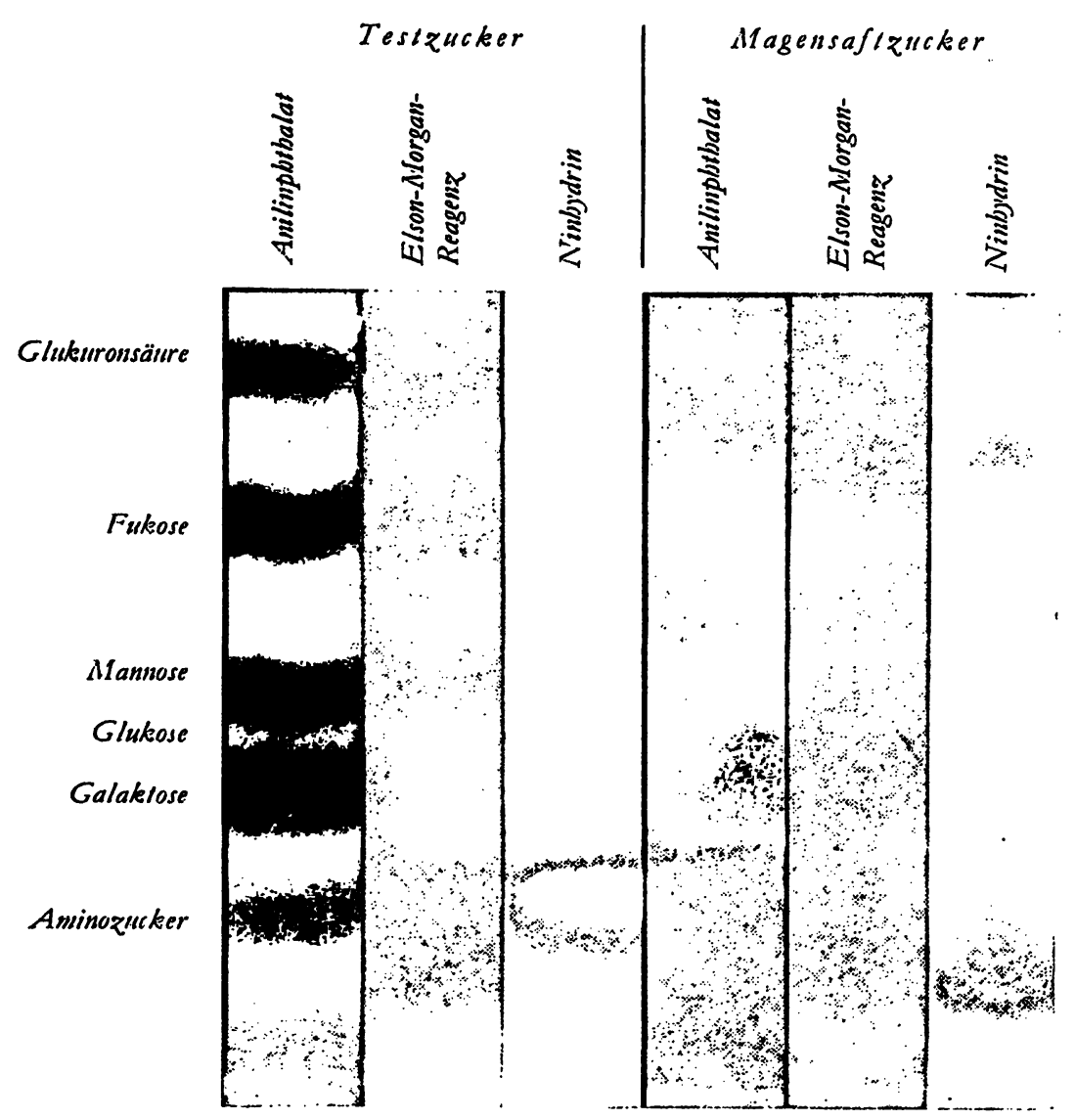

drin-Reaktion wurde die chromatographische Identität von Galaktosamin und Glukosamin festgestellt. Der Vergleich mit einem Standardzuckergemisch bekannter Zusammensetzung, das Galaktosamin, Glukosamin, Galaktose, Glukose, Mannose und Fukose enthielt, ermöglichte die qualitative Bestimmung der einzelnen Zucker, wobei $R_{F}$-Werte und Farbcharakter im sichtbaren Licht als Kriterien herangezogen wurden.

Dic halbquantitative Bestimmung erfolgte am Zeiss-Spektral-Photometer M $4 \mathrm{Q}$ mit Chromatogrammzusatz. Gemessen wurde bei einer Wellenlänge von $436 \mathrm{~m} \mu$ im durchfallenden Licht eines Quecksilberbrenners. Die entsprechend der Farbintensität der einzelnen Zonen im Streifen registrierte Absorption wurde als Funktion der jeweiligen Wanderungsgeschwindigkeit auf Papier übertragen und so für die weitere Auswertung fixiert. Nach Einzeichnen der Gaußsschen Kurven wurden ihre Flächenwerte planimetrisch ermittelt. Das Verhältnis der einzelnen Zuckerkomponenten zueinander wurde in relativen Prozenten angegeben; auf eine quantitative Auswertung wurde verzichtet.

\section{Ergebnisse}

Die Hydroly'se mit einem sauren Ionenaustauscher lieferte, verglichen mit der Hydrolyse durch konz. Salzsäure (Verhältnis Magensaft/konz. Salzsäure 2:1) über 2 Stunden bei $100^{\circ}$, bessere Ergebnisse. Bei der Säurehydrolyse traten eine Anzahl neuer unbekannter Fraktionen auf, die nicht identifiziert werden konnten. Bei der Ionenaustauscherhydrolyse fehlten derartige Fraktionen ganz oder traten nur in geringer Menge auf. Die quiantitative Ausbeute der beiden Hydrolyse-Verfahren unterscheidet sich nicht wesentlich.

Als günstigste Bedingungen für die Hochspannungselektrophorese wurde eine Spannung von $1750 \mathrm{~V}$ bei einer Trennzeit von 1,5 Stunden ermittelt. Hexosamine wandern kathodenwärts, Galaktose, Glukose, Mannose, Fukose und Glukuronsäure anodisch. Testzucker konn- ten nur unvollständig aufgetrennt werden. Außerdem traten, wie auch im biologischen Material, Banden auf, die sich an den Testzuckern nicht klassifizieren ließen.

Papierchromatographisch konnte mit der Anilinphthalatfärbung Galaktosamin, Glukosamin, Galaktose, Glukose, Mannose und Fukose nachgewiesen werden, dagegen keine Glukuronsäure. Versuche mit Testzuckern zeigten, daß die Werte der. berechneten rel. \% von Hexosamin, Galaktose, Glukose, Mannose und Fukose weitgehend konstant blieben (Abb. 1).

Für die Prüfung, ob Beziehungen zwischen dem histologischen Schleimhautbefund und dem Kohlenhydratspektrum des Magensaftes bestehen, wurden die bioptischen Schleimhautbefunde in normale und entzündlich veränderte gruppiert. Mit dem $\chi^{2}$-Test prüften wir die Unterschiede $z$ wischen den morphologischen Gruppen normale Magenschleimhaut und Gastritis hinsichtlich der Häufigkeit niedriger und hoher Werte für die Zucker des hydrolysierten Magensaftes. Aus den ermittelten rel. \%-Werten, bezogen auf die Gesamtzucker, wurde als Grenze der Mittelwert aus Minimal-Maximalwert gewählt. Für die Aminozucker ergab sich ein Bereich von $0-10$ rel. $\%$ und $11-20$. Die Häufigkeit dieser Befunde bei normaler Magenschleimhaut und chronischer Gastritis ist in Tabelle 1 wiedergegeben. Beziehungen $z$ wischen normaler Schleimhaut sowie Gastritis und den aus den Glykoproteiden des Magensaftes darstellbaren Zuckern Galaktose, Mannose, Fukose und Glukose konnten nicht nachgewiesen werden. 
Tab. 1

Aminozucker im Magensaftsekret

\begin{tabular}{|c|c|c|c|c|}
\hline & \multicolumn{2}{|c|}{$\begin{array}{c}\text { Relativer Wert in \% } \\
\text { bezogen auf Gesamtzucker } \\
0-10 \quad 11-20\end{array}$} & insgeșamt & in \% \\
\hline $\begin{array}{l}\text { Normale Magenschleimhaut } \\
\text { Chron. Gastritis } \\
\text { insgesamt } \\
\text { in } \%\end{array}$ & $\begin{array}{l}9(7,36) \\
9(10,64) \\
18 \\
81,82\end{array}$ & $\begin{array}{l}0(1,64) \\
4(2,36) \\
4 \\
18,18\end{array}$ & $\begin{array}{r}9 \\
13 \\
22 \\
100\end{array}$ & $\begin{array}{r}40,91 \\
59,09 \\
100,00\end{array}$ \\
\hline $\begin{array}{l}\text { Erwartete Häufigkeit } \\
\varphi_{i}\end{array}$ & $\mathrm{D}=\mathrm{z}_{\mathrm{i}}-\varphi_{\mathrm{i}}$ & $\mathrm{D}^{2}$ & & $D^{2}: \varphi_{1}$ \\
\hline $\begin{array}{r}7,36 \\
1,64 \\
10,64 \\
2,30\end{array}$ & $\begin{array}{r}1,64 \\
-1,64 \\
-1,64 \\
1,64\end{array}$ & $\begin{array}{l}2,69 \\
2,69 \\
2,69 \\
2,69\end{array}$ & & $\begin{array}{l}0,37 \\
1,64 \\
0,25 \\
1,14\end{array}$ \\
\hline & & & $\chi^{2}=3,40$ & \\
\hline
\end{tabular}

$Z \mathrm{i}=$ beobachtete Häufigkeit; $\varphi \mathrm{i}=$ erwartete Häufigkeit. $\chi^{2}=\sum_{\mathrm{i}=1}^{\mathrm{m}} \frac{(\mathrm{Zi}-\varphi \mathrm{i})^{2}}{\varphi \mathrm{i}}$

Ergebnis des $\chi^{2}$-Testes: Berechnet man aus den Randzahlen die bei Unabhängigkeit zu erwartenden Häufigkeiten - Werte in Klammern sowie $\varphi_{i}$ - so ergibt sich bei einem $\chi^{2}$ von 3,40 eine Irrtumswahrscheinlichkeit von etwas mehr als $5 \%$. Danach ist die Tendenz abzuleiten, daß bei Fällen mit chronischer Gastritis das Magensaftsekret einen höheren Aminozuckeranteil aufweist.

\section{Diskussion}

Die bei dieser Untersuchungsreihe und früheren Untersuchungen mit der Säurehydrolyse gefundenen unbekannten Fraktionen, die sich nicht identifizieren ließen, waren bei der Ionenaustauscherhydrolyse geringer ausgeprägt oder fehlten. Man darf annehmen, daß die Ionenaustauscherhydrolyse weniger sekundäre Umwandlungsprodukte liefert. Grob quantitative Unterschiede der beiden Hydrolyseverfahren bestehen jedoch nicht.

Die bisher bekannten Methoden zur Trennung von Zuckern mittels Hochspannungselektrophorese liefern nach den vorgelegten Resultaten keine befriedigenden Ergebnisse. Unvollständige Hydrolyse und mangelnde Reinigung von den bei der Hydrolyse entstandenen Spaltprodukten scheinen die Methode zu belasten. Die Zucker werden bei der Hochspannungselektrophorese im Boratpuffer zu Boraten verestert, wobei die Vorgänge im einzelnen noch wenig geklärt sind. In der Literatur mitgeteilte Resultate erstrecken sich nur auf Testzucker, nicht jedoch auf biologisches Material. Die vielen Unsicherheitsfaktoren lassen noch nicht zu, diese Methode zur Bestimmung der Kohlenhydratkomponenten des Magensaftes zu empfehlen.

Das Vorhandensein von Galaktosamin, Glukosamin, Galaktose, Glukose, Mannose und Fukose im Magensekret wurde bereits mitgeteilt $(1,2)$ und konnte bestätigt werden. Bei der Unterscheidung von normaler Magenschleimhaut und chronischer Gastritis zeigte sich, daß weder eine Zuckerkomponente excessiv vermehrt ist noch völlig fehlt. (Abb. 2 u. 3) Im Gegensatz zu den Arbeiten von Deutsch (7) ließen sich stets Mannose und Glu- kose in unterschiedlicher Menge nachweisen. Damit kann die Auffassung von DeutsCH, daß Mannose und Glukose nur bei einer Entzündung der Magenschleimhaut im Sekret erscheinen, nicht bestätigt werden. Als Ursache der Divergenz wäre die von Deursch benutzte Säurehydrolyse zu diskutieren, die uns für die Trennung der Zuckerkomponenten vom Proteinanteil nicht optimal erscheint. Glukuronsäure konnte nicht nachgewiesen werden, was offenbar methodisch bedingt ist. Entgegen der Ansicht von Schrager $(8,9)$ ist das Auftreten der

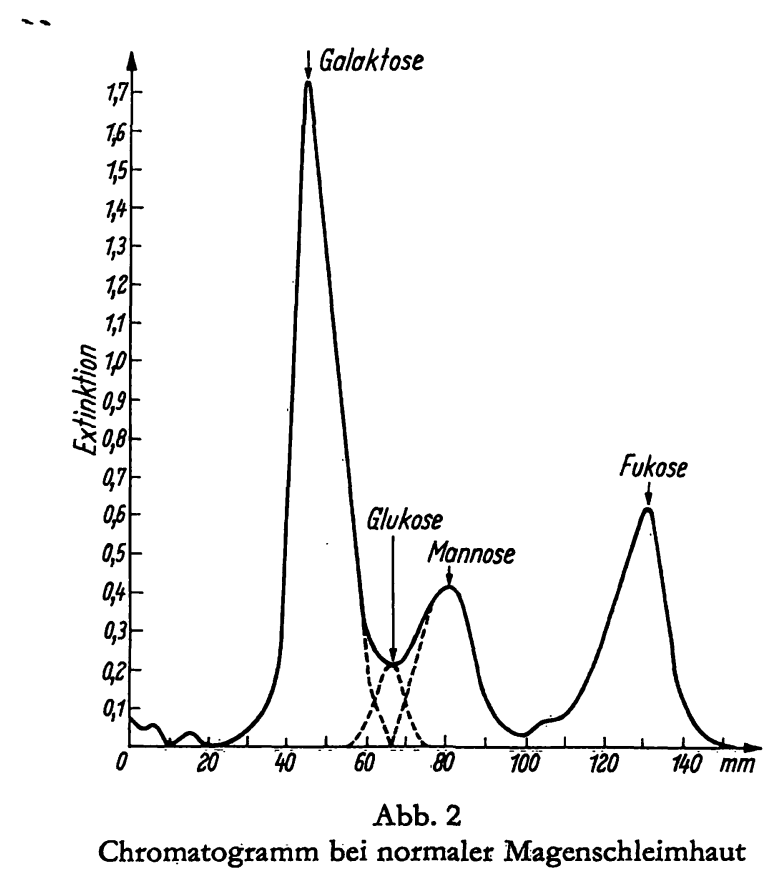

Hydrolyse und Chromatographie s. Legende Abb. 1. - Anilinphthalat-Reagenz; Auswertung im Spektralphotometer -. 


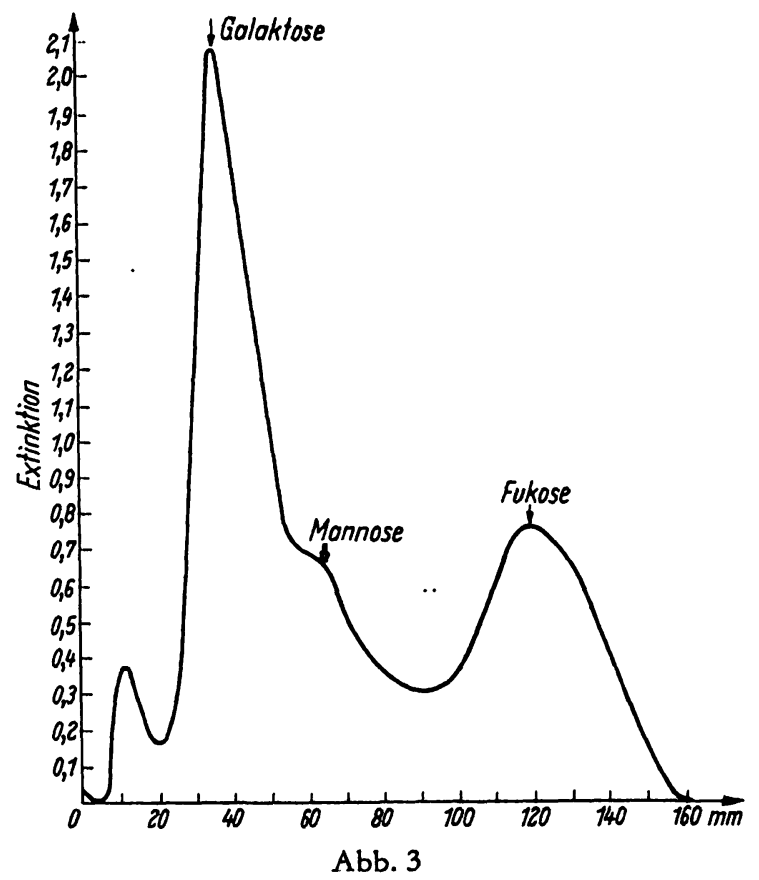

Chromatogramm bei atrophischer Gastritis

Hydrolyse und Chromatographie s. Legende Abb. 1. - Anilinphthalat-Reagenz; Auswertung im Spektralphotometer

Glukuronsäure, die sehr hitzeempfindlich ist, im menschlichen Magensaft von der Methode der Hydrolyse abhängig. Der Nachweis von Glukuronsäure gelingt, wenn man die chromatographisch als Glukuronsäure erkannte Bande mit Wasser eluiert und das Eluat nach Tolnens mit Naphthoresorcin und rauchender Salzsäure versetzt.

Quantitative Aussagen sind bei papierchromatographischen Analysen problematisch, da die verschiedenen Zuckerkomponenten sich nicht quantitativ anfärben. Unsere Ergebnisse bei einer Untersuchung von normaler Magenschleimhaut und Gastritis zeigen für den rel. $\%$-Gehalt der Aminozucker nach der $\chi^{2}$-Methode eine Tendenz, die nahezu die 5\%-Wahrscheinlichkeitsgrenze erreicht. Danach muß im Sekret bei normaler Magenschleimhaut ein niedrigerer Aminozuckergehalt erwartet werden, was sich auch mit den Befunden von Grass $(10,11)$ deckt. Einen erhöhten Aminozuckergehalt wird man bei einer chronischen Entzündung und vor allem bei der atrophischen Gastritis erwarten können. Eine verbesserte Methodik der Magensaftanalysen ist notwendig, um zu endgültigen Aussagen zu gelangen. Hinsichtlich der Aminozucker ist zu beachten, daß ein Teil der Hexosamine an den Ionenaustauscher quantitativ adsorbiert wird. Inwieweit Aminozucker sich durch Zugabe von Säure zum Magensafthydrolysat vom Ionenaustauscher eluieren lassen, um zu einem vollständig quantitativen Nachweis zu kommen, ist noch nicht restlos geklärt.

\title{
Literatur
}

1. Preisser, F., G. Berg und N. Henning, Zschr. Gastroenterol. 1, 155 (1963). - Berg, G. und F. Preisser, Gastroenterologia, Basel, Suppl. zu 97, 238 (1962). - 3. Berg, G., N. Henning, K. Heinkel und W. Lentzen, Klin. Wschr. 38, 282 (1960). 4. Clotren, R. und A. Clotren, Hochspannungselektrophorese, Georg Thieme Verlag, Stuttgart (1962). - 5. HAIs, J. M. und K. Macek, Handbuch der Papierchromatographie, Bd. I, Gustav Fischer Verlag, Jena (1958). - 6. CRAMER, F., Papierchromato- graphie, Verlag Chemie GmbH, Weinheim/Bergstr. (1962). 7. Deutsch, E., H. J. Christian und N. A. Peloquin, Amer. J. Gastroenterologie 41, 467 (1964). - 8. Schrager, J., Nature (London) 198, 899 (1963). - 9. Schrager, J., Nature (London) 201, 702 (1964). - 10. Glass, G. B. J., M. Rich und L. Stephanson, Gastroenterology, Baltimore 34, 598 (1958). - Glass, G. B. J., L. Stephanson-Liounis, M. Rich und S. E. Mitschell, Proc. Wld. Congr. Gastroenterol. 1958 994, Baltimore.

Privatdozent Dr. med. G. Berg

Med. Univ.-Klinik, 852 Erlangen, Krankenhausstr. 12

\section{Die Fraktionierung von Pleurapunktat-Proteinen im Zusammenhang mit der Bestimmung von Entzündungsaktivitäten}

\author{
Von J. WAGNER und B. SCHMIDT
}

Aus der Medizinischen Klinik der Karl-Marx-Universität Leipzig (Direktor: Prof. Dr. R. Emmrich)

Herrn Prof. Dr. Dr. h. c. Dr. h. c. Dr. h. c. Dr. h. c. K. Mothes zum 65. Geburtstag

(Eingegangen am 23. August 1965)

\begin{abstract}
Die Pleurapunktat-Proteine wurden mit verschiedenen „Sephadex“-Typen fraktioniert, wobei mit Sephadex A-25 6 verschiedene Fraktionen erhalten wurden. Die mit dem Trypanblau-Test durchgeführten Bestimmungen der Entzündungsaktivität waren in den Fraktionen positiv, die neben Albumin $\alpha_{1^{-}}$und $\alpha_{2}$-Globuline enthielten. Diese Ergebnisse konnten durch eine fraktionierte Fällung mit Ammoniumsulfat bestätigt werden.

The proteins from pleural puncture were fractionated with various types of Sephadex; six fractions were obtained with Sephadex A-25. Tests for inflammatory activity with trypan blue were positive for those fractions which ran close to albumin, $\alpha_{1}$-and $\alpha_{2}$-globulins. These results were confirmed by fractionation with ammonium sulphate.
\end{abstract}

Die Exsudation bei der akuten Entzündung wird nach LEwIs (1) hauptsächlich durch Freisetzen der in den Mastzellen befindlichen stark vasoaktiven Stoffe hervorgerufen. Relativ geringe mechanische, thermische, elek- trische und aktinische Reize führen zu einer sofortigen Entgranulierung dieser Zellen mit Abgabe ihrer Inhaltsstoffe. Neben dem Histamin und dem Heparin spielen seit einigen Jahren kreislaufwirksame Polypep- 